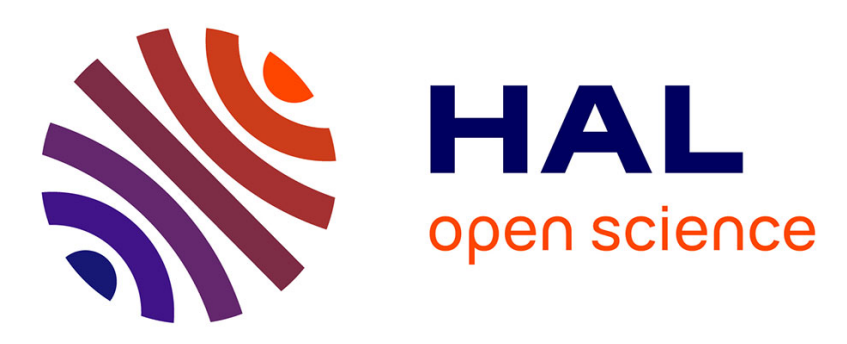

\title{
An Anthropomorphic Navigation Scheme for Dynamic Scenarios
}

\author{
Leonardo Scandolo, Thierry Fraichard
}

\section{To cite this version:}

Leonardo Scandolo, Thierry Fraichard. An Anthropomorphic Navigation Scheme for Dynamic Scenarios. ICRA 2011 - IEEE International Conference on Robotics and Automation, May 2011, Shanghai, China. pp.809-814, 10.1109/ICRA.2011.5979772 . inria-00563693

\section{HAL Id: inria-00563693 https://hal.inria.fr/inria-00563693}

Submitted on 7 Feb 2011

HAL is a multi-disciplinary open access archive for the deposit and dissemination of scientific research documents, whether they are published or not. The documents may come from teaching and research institutions in France or abroad, or from public or private research centers.
L'archive ouverte pluridisciplinaire HAL, est destinée au dépôt et à la diffusion de documents scientifiques de niveau recherche, publiés ou non, émanant des établissements d'enseignement et de recherche français ou étrangers, des laboratoires publics ou privés. 


\title{
An Anthropomorphic Navigation Scheme for Dynamic Scenarios
}

\author{
Leonardo Scandolo and Thierry Fraichard
}

\begin{abstract}
This paper is concerned with the navigation of personal robots in human-populated environments. The behavior of a person among its peers is governed by a number of unspoken social rules, e.g. maintaining an appropriate distance. The primary contribution of this paper is a navigation scheme that is anthropomorphic, i.e. that emulates human behaviors and seeks to adhere to these social rules. Unlike previous works in this area, the focus herein is on dynamic scenarios. The navigation scheme proposed explicitly reasons on the future behavior of the people involved so as to produce better socially acceptable trajectories (not to mention safer trajectories as well). The navigation scheme relies upon a novel cost function called the social costmap that captures in a unified way the different social rules imposed by the people populating the robot's workspace.
\end{abstract}

\section{INTRODUCTION}

As personal robots become more common, their behavior needs to be refined in order to meet the expectations of the general population. Over generations, humans have developed a form of non-verbal communication that uses space as its medium. This is the realm of proxemics, which is the area of study of communication through the use of space, body positions and proximity [1], [2]. Although these social rules are not explicit, people feel general discomfort when they are broken [3]; such is the case when a person stands too close to another one, or when he turns his back to an interlocutor. Since it has been shown that people behave towards personal robots the way they would towards human [4], such rulebreaking behaviors will cause a similar discomfort when performed by robots, so it is imperative that the algorithms that control robots interacting with humans be designed to emulate the expected behaviors of humans. In other words, they should yield socially acceptable behaviors so as to facilitate the integration of robots in human-populated environments and their acceptance.

This paper is concerned with the navigation of personal robots in human-populated environments (Fig. 1-top). The goal is to design a navigation scheme that is anthropomorphic, i.e. that emulates human behaviors and adheres to the unspoken social rules that govern the motion of a person among its peers. The introduction of social rules in navigation is fairly recent but interesting results have already been obtained (see $\S \mathrm{II}$ ). In most cases works in this area do not fully take the time dimension into account in the motion planning process; they usually make a "frozen-world" assumption and rely instead on fast on-line recalculation of the planned motions to handle the changes in the environment. However, from a motion safety point of view, the

INRIA, LIG-CNRS and Grenoble University (FR).

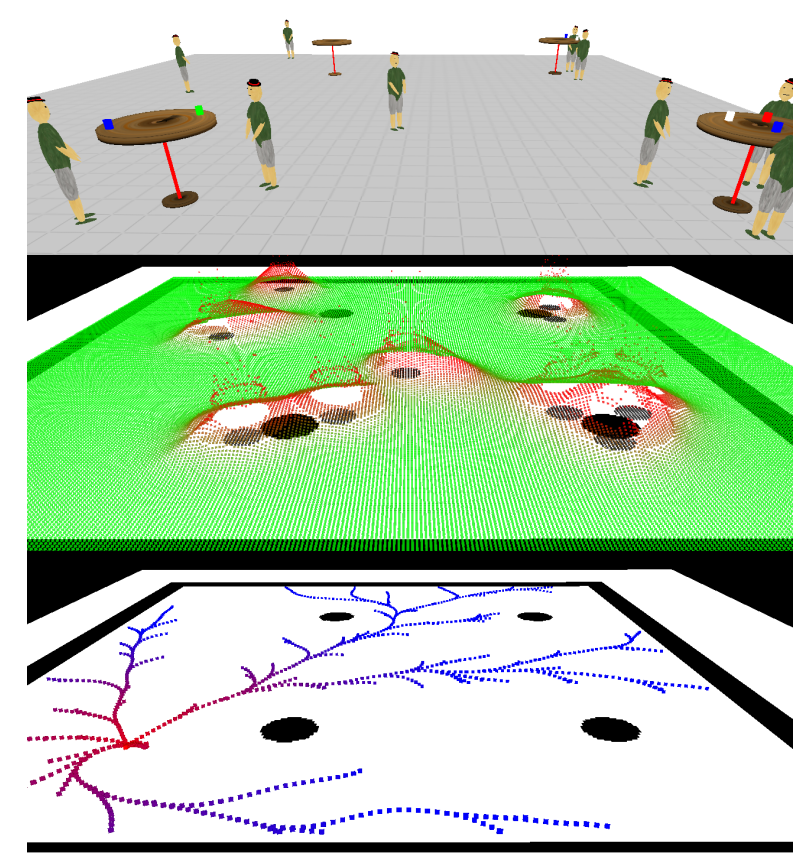

Fig. 1. A human-populated environment (top); the corresponding social costmap (middle); a trajectory planning tree (bottom).

analysis done in [5] has shown the flaw in this assumption and the importance of explicitly reasoning about the future evolution of the environment in order to guarantee collision avoidance. It turns out that this is also true from a behavioral point of view: it will be shown that better anthropomorphic behaviors can be obtained by reasoning about the future evolution of the environment. The focus of the paper is therefore on dynamic scenarios. The primary contribution is an anthropomorphic navigation scheme aimed at dealing with dynamic environments.

To begin with, the social rules that should be obeyed by the robot are identified. These rules are derived from works in the fields of environmental psychology (and more specifically proxemics), and experiments carried out to test the reactions of people to different behaviors displayed by robots. Different rules identified in those works lead to different constraints, from which different mathematical models are derived. The first contribution of the paper is an integrated model of these different rules. It takes the form of a cost function defined on the robot's workspace. This cost function is called social costmap (SCM), it captures in a unified way the social rules imposed by the different people (whether moving or not) populating the robot's workspace 
(Fig. 1-middle).

The second contribution of the paper is the anthropomorphic navigation scheme itself. It follows the Partial Motion Planning paradigm (PMP), a compromise between purely reactive navigation and full-fledged motion planning [6], designed to take into account the various constraints imposed by dynamic environments: upper-bounded planning time and motion safety. At each navigation cycle, PMP builds a tree in the state-time space of the robot and returns the best possible trajectory (Fig. 1-bottom). In the context of this paper, PMP uses the information about the future evolution of the environment and the corresponding SCMs to determine, at each navigation cycle, the best socially acceptable trajectory.

The paper is organized as follows: the relevant literature in both Environmental Psychology and Robotics is reviewed in $\S$ II. The social costmap model is presented in $\S I I I$. The anthropomorphic navigation scheme is detailed in $\S I V$. Finally, experimental results obtained in simulation are presented in $\S \mathrm{V}$.

\section{RELATED WORKS}

\section{A. Environmental Psychology}

The field of environmental psychology has been around since the second half of the $20^{\text {th }}$ century. It attempts to understand how a person's behavior and feelings are affected by its surroundings. We are interested specifically in the way the presence and actions of other people affect an individual, that is to say, the effects of the social environment. This is because it is important for a robot that needs to move in a social environment to try to minimize the discomfort it inflicts on the people around it by behaving in a way similar to that of a human, since experiments have shown that people behave around robots as they do around other people [4].

An important area of environmental psychology for this work is thus proxemics, which deals with the way people interact with each other through the use of space. The idea that a person may have a notion of personal space which is around himself and is a cause of discomfort when other people trespass it dates from the 1950's [1]. Edward T. Hall coined the word proxemics by combining the words proximity and semiotics. He wanted to convey the idea of people communicating by the use of space and other physical factors [2].

One of the first papers to delve into the matter of proxemics is [2]. In it, Hall describes a system for classifying different types of proxemic behavior, such as face orientation, proximity, voice loudness and posture among others. The system is fairly simple and oriented towards instructing an anthropologist on what cues to give attention to while observing a social interaction; nevertheless it is very useful for understanding how to model non-verbal communication and unspoken social rules for close interaction.

Since then, experiments have been carried out to try to best define the notion of personal space and to assess its size and the reaction of people to it being violated. In [3], Middlemist et al. conducted an experiment that confirms that violation of personal space is a cause of arousal, which leads to discomfort. Kaya and Erkíp in [7] show how personal space changes in size and shape in crowded areas and also depending on the sex of the person. In [8] and [9] an overview of the field of environmental psychology and non verbal communication can be found. The author of [8] cites numerous results on the field, which lead him to make some observations on the subject of personal space, its shape, and factors that may affect it. Some of the conclusions in his book are that personal space is mostly circular (if viewed from above) and slightly bigger on the front. Also, the shape and size of a person's personal space are different depending on the person's race and culture. He also observes that in crowded spaces, people tend to disregard personal space, and treat other people as they would objects.

Other more subtle factors also play a role in the field of environmental psychology. In [10], the reaction of people to different types and amounts of eye contact are analyzed, as well as the way it influences other behaviors. Argyle, in [8], analyzes a lot of different factors that take part in social interactions, such as posture, gaze direction, gestures, physical contact and smell among others. In [11], Sisbot et al. proposes and tests a general human aware navigation scheme. In it, humans are modeled by describing their structure and kinematics, including their position and orientation, and information needed to compute costs depending on the person's geometric configuration and pose. Taking these variables into account, several criteria are used to construct potential fields in a $2 \mathrm{~d}$ grid in which the space is divided, and then merge them into a single grid that the planner uses to calculate the optimum plan for a given situation. An $A^{*}$ algorithm is used to find the best route between two points in the grid. It is assumed in the work that any person in the environment is static, and it relies on fast recalculation time should a person move.

\section{B. Robotics}

In [12] a model is presented to attempt to mimic the behavior of a human while joining, maintaining and leaving a group conversation. Its input is limited to the location and orientation of humans in the environment. With this information, a robot regulates its velocity in order to maintain a constant distance with a person, and an orientation that allows it to face him. For groups of people an average of the velocity and orientation is computed to ensure that the robot faces the middle of the group and stays within a coherent distance of all the participants.

Amaoka et al. in [13] propose a mathematical model of personal space in order to apply it to agents in virtual worlds and simulate realistic behavior. They use two 2dimensional Gaussian functions which represent the personal space around an individual. They then test a very simple navigation scheme using this information.

In [14], a navigation planner is presented for different tasks. It works by applying an $A^{*}$ algorithm over a variable size 2-dimensional grid. The humans and the robot in the environment are modeled by their pose and velocity, and the planner takes into account the expected result of an action on 
the configuration of the robot and the expected change in the position of a person to assess the fitness of a possible robot action. The planner assumes that there will be no change in the velocity of any of the people in the environment, and relies on fast on-line recalculation should a change occur.

In [15], human safety for the use of high-speed robots is enforced through the use of a velocity limit that is dependent on the distance to a human. The authors ran experiments and concluded that a linear relation exists between velocity and distance that ensures that people in the robot's environment do not experience any discomfort or uncertainty because of the robot's motions.

Lastly, the work in [16] very closely resembles the one presented in this paper; it was published after the initial submission of this work and brought to our attention by our reviewers. The authors of that work present an approach that builds a cost map of the environment and arrive at a trajectory building upon an RRT planner. The main differences between the two works reside in the definition of the cost map, as well as in the actual use of the cost map during the planning stage. While they simply sample the configuration-time space and expand using a typical RRT scheme, and then choose the best path based on the cost map, the work presented here takes the cost map into account during the tree creation, thus biasing the paths created towards more socially acceptable ones.

\section{Social Costmap Model}

\section{A. Environment}

The model of the world presented in this section will be 2-dimensional, meaning that the workspace $\mathbb{W}$ will be equivalent to $\mathbb{R}^{2}$.

The positions and vectors specified will be given for a common fixed frame of reference.

1) People: A human in the environment will be modeled as a tuple $p=\left(p_{x}, p_{y}, p_{\theta}, p_{v}, p_{r}\right)$, where:

- $\left(p_{x}, p_{y}\right) \in \mathbb{R}^{2}$ is the position of the person.

- $p_{\theta} \in \mathbb{S}$ is the gaze direction of the person

- $p_{v} \in \mathbb{R}^{2}$ is the linear velocity of a person

- $p_{r} \in \mathbb{R}$ is the radius of the disk that represents the person.

2) Objects in the environment: The information about the obstacles in the environment will also be present in order to avoid plans that will lead to a collision. In this case an object $\mathcal{B}$ can have an arbitrary geometric shape and location. Therefore the environment will also contain $n$ objects $\mathcal{B}_{1}, \ldots, \mathcal{B}_{n}$ where $\mathcal{B}_{i} \subset \mathcal{W} \forall i$.

3) Interaction sets: One of the goals of the planning algorithm is to avoid trajectories where the robot blocks the vision of a person with respect to the entity or entities he is interacting with. These can be either objects or people in the environment. Therefore a list of interaction sets which can contain people and objects in the environment will be available. These sets will indicate the assumption that all the entities in it are interacting with each other, either actively or passively. The space between two interacting entities will be referred to as an interaction region.
4) Social environment: The social environment of the robot will consist of a set of people $p s=\bigcup p$, a set of objects $\mathcal{B} s=\bigcup \mathcal{B}$ and the set of all interaction sets $i s$. Therefore, besides the information about the robot that is being planned for, the input of the planning algorithm will consist of the social environment $\mathcal{S}=\{\mathcal{B} s, p s, i s\}$.

\section{B. Constraints}

The social costmap $(S C M)$ will take as input the social environment $\mathcal{S}$ and map points in the workspace $\mathcal{W}$ to a cost value in the range $[0, \infty]$; lower values representing lower discomfort caused by a robot occupying that point in space. It will be defined as an aggregate of different cost functions that arise from constraints imposed by social behavior expectations.

1) Static Personal Space: Personal space is a very important concept, and it is central to Hall's proxemic theory. In his seminal works, such as [2], he proposes the idea of different spaces of interaction for humans, which are concentric rings around an individual. He refers to them, in increasing size order, as intimate, personal, social and public spaces. Unwanted invasion of a person's personal space is cause of discomfort and anxiety, even more so for invasion of the intimate space. Hall's concept of interaction spaces of a person will be modeled as a costmap whose costs will be symmetrical around him. The cost function regarding a person $p$ for a point $r=\left(r_{x}, r_{y}\right)$ in this case will be $P S$ defined as:

$$
P S(p, r)=\left\{\begin{array}{cl}
\infty & \text { if } d \leq p_{r} \\
\frac{1}{w_{i}\left(d-p_{r}\right)}+w_{I} & \text { if } p_{r}<d \leq d_{i} \\
\left(w_{I}-w_{P}\right) e^{-\left(\frac{d-d_{i}}{2 w_{p}^{2}}\right)} & \text { if } d_{i}<d \leq d_{p} \\
w_{p} e^{-\left(\frac{d-d_{p}}{2 c_{s}^{2}}\right)} & \text { if } d_{p}<d
\end{array}\right.
$$

Where $d=\operatorname{dist}(p, r)$ is the distance between point $r$ and the center of the circle by which person $p$ is modeled. Parameter distances $d_{i}$ and $d_{p}$ represent the intimate space radius and the personal space radius respectively. The slopes of the function are defined by the weight parameters $w_{i}, w_{p}$ and $w_{s}$.

2) Back Space: In general, a human is most comfortable when he is aware of all the moving objects or people in his environment. Therefore it is sensible to censor trajectories that travel closely behind a person because they stand to cause fear or discomfort. Psychological works such as [3] provide empirical evidence of this behavior. This constraint attempts to minimize that discomfort by assuming a blind angle of $\alpha$ radians behind an individual, and giving the space directly inside that region a high cost value, which will decrease the further away the point is, anda the smaller the angular distance with the gaze line. The cost function will only take non-zero values inside back space region of angle $\alpha$. The vector $j=-p_{\theta}$ is defined, and is the inverse vector from the person's gaze vector, which points in the direction 
of the blind zone. The vector $v=\left(p_{x}, p_{y}\right)-\left(r_{x}, r_{y}\right)$ is also defined. The back space function $B S$ will be defined as:

$$
B S(p, r)=\left\{\begin{array}{cl}
0 & \text { if } \angle(j, v)>\frac{\alpha}{2} \\
w_{m} \beta(g, r) e^{-\left(\frac{|d|^{2}}{2 w_{s}^{2}}\right)} & \text { otherwise }
\end{array}\right.
$$

Again $d$ is the distance between point $r$ and person $p$. The weight parameter $w_{m}$ controls maximum value of the cost of the function and the weight parameter $w_{s}$ controls its slope. The function $\beta(g, r)$ provides a weight for the angular distance between the gaze direction and the vector $v$.

3) Personal Space during Motion: During motion the personal space of a person is enlarged as a natural safety measure to avoid coming close to an obstacle. This ensures that the person has enough time to maneuver should something unexpected happen. The study performed in [17] found that personal space during motion resembles an ellipse.The motion space (MS) costmap will be defined in order to capture the constraints imposed by this behavior. The point $\left(c_{x}, c_{y}\right)$ is defined as the projection of the point $r=\left(r_{x}, r_{y}\right)$ into the reference frame whose origin is $\left(p_{x}, p_{y}\right)$ and whose positive $y$ axis is aligned with the subject's velocity vector $\left(p_{v}\right)$. The definition of the personal space function during motion follows:

$$
M S(p, r)=\left\{\begin{array}{cl}
w_{m} e^{-\left(\frac{c_{x}^{2}}{2 w_{m x}^{2}}+\frac{c_{y}^{2}}{2 w_{m y}^{2}}\right)} & \text { if } c_{y}>0 \\
0 & \text { otherwise }
\end{array}\right.
$$

In this case $w_{m x}$ and $w_{m y}$ define the ellipsis' slope and size so they are dependent on $p_{v}$, such that for an average walking velocity the costmap that is formed will be of a size according to the findings of [17]. As it stands, $w_{m x}$ and $w_{m y}$ can (and should) have different change rates. $w_{m}$ is a parameter that defines the maximum height of this cost function.

4) Merging the different constraints: A unified model of the personal space constraints needs to be defined, contemplating the concepts of static personal space, back space and personal space during motion. Therefore the dynamic personal space (DPS).

The DPS will be defined in a way that will ensure that static personal space is always preserved, but when a subject starts walking, his personal space will morph accordingly.The DPS costmap will be defined simply as:

$$
D P S(p, r)=\max (B S(p, r), M S(p, r), P S(p, r))
$$

One parametrization of the DPS costmap is shown in Fig. 2, for a human moving forward at a velocity assumed to be the normal walking speed of an individual.

5) Interaction regions: The purpose of this constraint can be simply stated as "Do not traverse the space between two interacting entities." Behavioral experiments such as [18] attest that unwanted interruption of visual connection between interacting entities is cause of discomfort.

For every interaction set $i$ in $\mathcal{S}$, we will model this constraint as a cost function defined as follows:

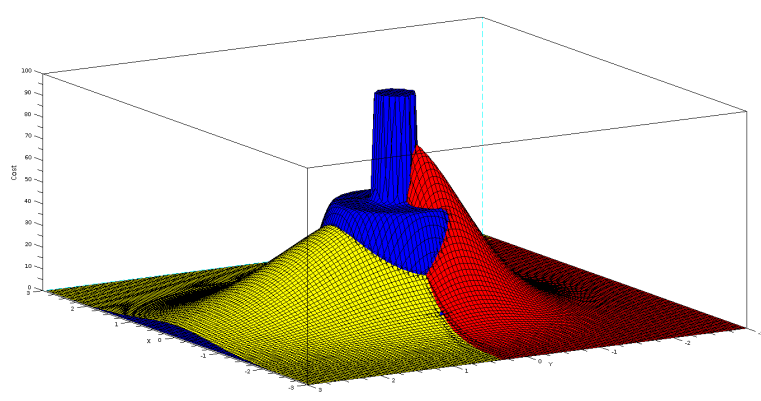

Fig. 2. Dynamic Personal Space

- For the case where there are only 2 elements in the interaction set $i$, namely $e_{1}$ and $e_{2}$, the whole region between them will be considered as a high cost area. That area will be defined as the convex hull of $e_{1} \cup e_{2}$. Formally, this cost function will be modeled as:

$$
\operatorname{IR}(i, r)= \begin{cases}w_{m} & \text { if } r \in \operatorname{ch}\left(e_{1}, e_{2}\right) \\ 0 & \text { otherwise }\end{cases}
$$

Where $\operatorname{ch}\left(e_{1}, e_{2}\right) \subset \mathbb{R}^{2}$ is the convex hull of $e_{1} \cup e_{2}$, and the weight parameter $w_{m}$ defines the cost of the interaction region.

- For the case of 3 elements or more in $i$, the point $c_{p}$ is defined as the barycenter of all the positions of the elements. The interaction region for the set $i$ will be defined as the union of the interaction regions defined between the elements of $i$ and an interaction focus $i f$. The region occupied by if will be a circle with center point $c_{p}$, and whose radius $w_{r}$ is a parameter of the cost function. Formally, the interaction region cost is defined as:

$$
I R(i, r)= \begin{cases}w_{m} & \text { if } \exists e \in i \cdot r \in \operatorname{ch}(e, i f) \\ 0 & \text { otherwise }\end{cases}
$$

Where again $w_{m}$ defines the cost of the interaction region.

6) Merging all the constraints: The social costmap cost function will be defined as the summation of the costmaps we have defined previously, this is modeled this way so as to give higher costs to areas in which a higher amount of people are being disturbed, thus ensuring that the least people are discomforted by the chosen trajectory. The social costmap is therefore defined as:

$$
\operatorname{SCM}(\mathcal{S}, r)=\sum_{p \in \mathcal{S}} \operatorname{DPS}(\mathcal{S}, p, r)+\sum_{i \in \mathcal{S}} I R(i, r)
$$

\section{ANThropomorphic NAVIGATION SCHEME}

\section{A. Partial motion planning}

Given the dynamicity of an environment populated with people, a navigation scheme needs to comply with real time constraints in order to ensure safety conditions. That is why we chose to implement a partial motion planning (PMP)[6] scheme that makes use of the social cost map.

This scheme iterates allowing a lower level planner create a map for a limited time. Once the time is up, it takes the first steps of the best trajectory found so far and concurrently 

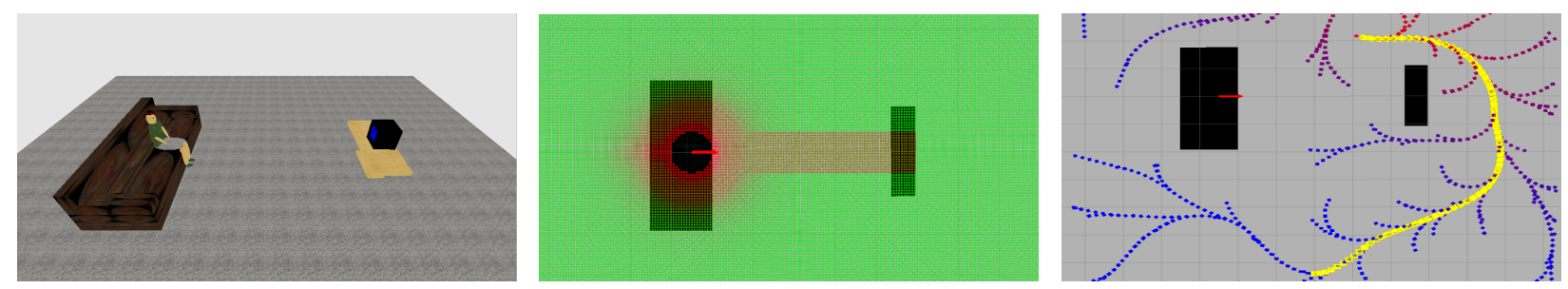

Fig. 3. A house scenario (left), the social costmap mapped (middle) and an iteration of the planner (right).

executes those steps while allowing the lower level planner to continue calculating a best trajectory with updated information about the environment. This ensures that the planner is responsive and works with up-to-date data on its surroundings.

\section{B. Building the tree}

For the lower level planner which builds a tree to explore the state-time space, an RRT [19] method was chosen.

At each iteration, this method picks a random configuration and expands the closest node to it towards it. This ensures a rapid coverage of the configuration space, ensured by the randomness of the configuration chosen at every step.

The algorithm implemented slightly modifies the way in which a regular RRT planner works by making use of the SCM: every time a new node is expanded its SCM value is computed and stored; when the algorithm needs to choose the nearest neighbor to expand, and the action control to apply, the metric used is biased towards less costly states, ensuring that states which cause discomfort on people are less frequently expanded.

\section{Choosing a trajectory}

Once the time to expand the tree has expired, a branch of the tree must be chosen that will provide a trajectory for the robot to follow. The selection of this branch is in this case biased towards the least costly trajectories in terms of the social cost function. All of the nodes in the tree are assessed and the one that provides the best compromise between a defined metric for the configuration space of the robot and the aggregated cost of its branch is selected for execution.

\section{Simulation Results}

\section{A. Simulation}

The planner was implemented within a $\operatorname{ROS}^{1}$ based navigational architecture. Within this architecture, it consists of a node which receives information about the expected behavior of the people in its environment and the static obstacles in it; it then iterates providing an incremental plan which is executed by another component in the ROS architecture, and its behavior is simulated in the 3D simulator Gazebo $^{2}$ in order to assess the characteristics of the plans returned by the planner.

\footnotetext{
${ }^{1}$ www.ros.org

${ }^{2}$ www.playerstage.sourceforge.net
}

\section{B. Robot model}

A differential drive monocycle system was used for the experiments. Therefore the states of the robot $\mathcal{A}$ consist of a tuple $s=(x, y, \theta, v, \omega)$ where $(x, y)$ is the position of the robot, $\theta$ its orientation, $\mathrm{v}$ its linear velocity and $\omega$ its angular velocity.

A control action for the model is a tuple $u=(\sigma, \epsilon)$ where $\sigma$ is the linear acceleration and $\epsilon$ the angular acceleration.

The differential equations for the model follow:

$$
\begin{aligned}
& \dot{x}=v \cos \theta \\
& \dot{y}=v \sin \theta \\
& \dot{\theta}=\omega \\
& \dot{v}=\sigma \\
& \dot{\omega}=\epsilon
\end{aligned}
$$

Where the acceleration and velocity values have upper and lower bounds.

\section{Results}

The planner was tested in several scenarios, which provide distinct difficulties that the planner should overcome graciously. They included bar and factory settings, open areas such as parks or streets, and more space restricted settings such as a hallway, an elevator and an office entrance.

The scenarios provided great input in order to fine tune the cost model. Once that phase was completed, it was successful at navigating through all the situations that were created.

Fig. 3 shows the house scenario.The SCM is plotted in a grid, with red points indicating high cost areas.The plan that is chosen in one of the iterations of the algorithm is highlighted in the last frame, among the tree that is built for that time window.

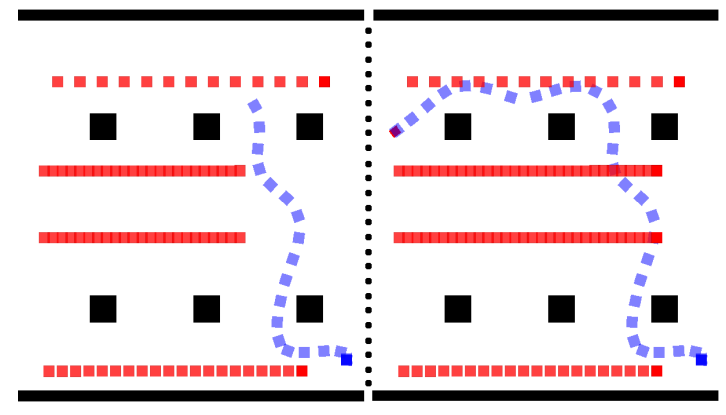

Fig. 4. Sequence depicting the trajectory chosen by the planner in the factory scenario in order to avoid crossing the paths of incoming people 


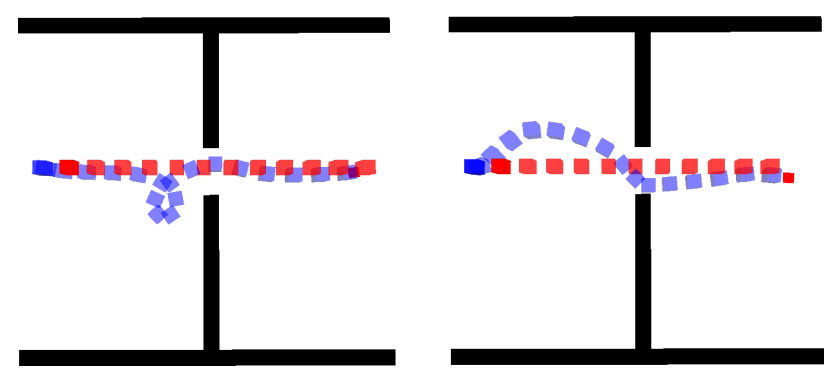

Fig. 5. Difference between the plans outputted by the planner when only state space is explored (left) and when state-time space is explored (right)

TABLE I

AVERAGE EXECUTION TIMES FOR DIFFERENT STEPS OF THE PLANNER

\begin{tabular}{|l|r|}
\hline & Exec. Time \\
\hline SCM cost calculation & $<10 \mu \mathrm{s}$ \\
\hline RRT iteration & $0.1 \mathrm{~ms}$ \\
\hline Finding best plan & $0.16 \mathrm{~ms}$ \\
\hline
\end{tabular}

Fig. 4 shows the trajectory the planner arrived at in a situation where several people are coming towards the robot. In this case a plan was chosen that stays away from incoming people as much as possible by timing its passing through different corridors in order not to run into any human.

Fig. 5 depicts the different trajectories arrived at by both the planner and a modified version of it that works only in state space for the door crossing scenario. In it it is clearly visible the increase in quality of the trajectries taken by the planner when the time dimension is taken into account.

Table I shows average computing times for several parts of the trajectory planner. The results were obtained on a laptop with an Intel Core2 Duo running at $2.8 \mathrm{GHz}$. The amounts of nodes tested and added to the tree per PMP cycle averaged between 2100 and 2800 for a time window of half a second. The SCM cost calculation is fast enough not to produce any noticeable performance change as the number of people in the environment varies.

\section{CONClusions AND Future Work}

An anthropomorphic navigation scheme has been presented that utilizes information about its environment to compute trajectories that are socially acceptable. This navigation scheme is based on a Partial Motion Planning architecture. At its core it utilizes a modified version of an RRT algorithm that uses costmap information to bias the tree expansion towards less costly trajectories. The costmap is obtained from a model of acceptable human spatial behavior, which is supported by empirical data obtained in the field of proxemics, and on human-robot interaction experiments.

The navigation scheme was successfully tested in a simulated environment to assess the quality of the approach; several scenarios with unique difficulties were tested to make sure the approach is sound and the plans reached respected the basic principles of human proxemics.
Future work lies in the direction of imposing stricter safety constraints into the planner, such as adding an inevitable collision states checker [20]. Another line of work could lie in exploring different ways of using the SCM described, such as implementing the techniques found in [21]. Other future works include extending the model to allow for more complex interactions, such as following a person, or handing over objects, and using a flocking simulator such as [22] to better predict human motion in a real environment.

Finally, testing this planning scheme on a robotic platform in a real life situation is a pending line of work.

\section{REFERENCES}

[1] R. Sommer, "Studies in personal space," Sociometry, vol. 22, 1959.

[2] E. Hall, "A system for the notation of proxemic behaviour," American Anthropologist, New Series, vol. 65, no. 5, October 1963, selected papers in Method and Technique.

[3] R. Middlemist, E. Knowles, and C. Matter, "Personal space invasions in the lavatory: suggestive evidence for arousal." Journal of personality and social psychology, vol. 33, no. 5, May 1976.

[4] L. Takayama and C. Pantofaru, "Influences on proxemic behaviors in human-robot interaction," in IEEE/RSJ Int. Conf. Intelligent Robots and Systems. IEEE Press, 2009.

[5] T. Fraichard, "A short paper about motion safety," IEEE Int. Conf. Robotics and Automation, 2007.

[6] S. Petti and T. Fraichard, "Partial Motion Planning Framework for Reactive Planning Within Dynamic Environments," in IFAC/AAAI Int. Conf. Informatics Control, Automation and Robotics, Barcelona (SP), Sept. 2005.

[7] N. Kaya and F. Erkip, "Invasion of personal space under the condition of short-term crowding: A case study on an automatic teller machine," Journal of Environmental Psychology, vol. 19, no. 2, 1999.

[8] M. Argyle, Bodily communication. Methuen \& Co, 1988.

[9] D. Nagar, Environmental psychology. New Dehli: Concept Publishing Company, 2006.

[10] M. Argyle and J. Dean, "Eye-contact, distance and affiliation," Sociometry, vol. 28, no. 3, September 1965.

[11] E. A. Sisbot, "Towards human-aware robot motions," Ph.D. dissertation, Universit Paul Sabatier, Toulouse (FR), Oct. 2008.

[12] P. Hiroshi, I. Hiroshi, K. Takayuki, M. Takahiro, and H. Christensen, "Navigation for human-robot interaction tasks," in IEEE Int. Conf. Robotics and Automation, 2004.

[13] T. Amaoka, H. Laga, and M. Nakajima, "Modeling the personal space of virtual agents for behavior simulation," in Int. Conf. CyberWorlds, Washington, USA, 2009.

[14] R. Kirby, "Social robot navigation," Ph.D. dissertation, Robotics Institute, Carnegie Mellon University, Pittsburgh, PA, May 2010.

[15] D. Shi, E. Collins, A. Donate, X. Liu, B. Goldiez, and D. Dunlap, "Human-aware robot motion planning with velocity constraints," in Int. Symp. Collaborative Technologies and Systems, May 2008.

[16] M. Svenstrup, T. Bak, and H. Andersen, "Trajectory planning for robots in dynamic human environments," in IROS 2010, 2010, pp. $4293-4298$.

[17] M. Grin-Lajoie, C. Richards, and B. McFadyen, "The negotiation of stationary and moving obstructions during walking: anticipatory locomotor adaptations and preservation of personal space." Motor control, vol. 9, no. 3, July 2005.

[18] M. Argyle and J. Graham, "The central europe experiment: Looking at persons and looking at objects," Journal of Nonverbal Behavior, vol. 1, no. 1, September 1976.

[19] J. Kuffner, J.J. and S. LaValle, "Rrt-connect: An efficient approach to single-query path planning," in IEEE Int. Conf. Robotics and Automation, 2000.

[20] T. Fraichard and L. Martinez-Gomez, "Ics-checker: an efficient and generic $2 \mathrm{~d}$ inevitable collision state checker," IEEE TRANS. ON ROBOTICS (submitted), 2010.

[21] L. Jaillet, J. Cortes, and T. Simeon and, "Sampling-based path planning on configuration-space costmaps," IEEE Transactions on Robotics, vol. 26, no. 4, August 2010.

[22] S. J. Guy, J. Chhugani, S. Curtis, P. Dubey, M. Lin, and D. Manocha, "Pledestrians: a least-effort approach to crowd simulation," in 2010 ACM SIGGRAPH/Eurographics, 2010, pp. 119-128. 